See discussions, stats, and author profiles for this publication at: https://www.researchgate.net/publication/350101280

\title{
Sympathy for the underdog: people are inclined to adopt the emotional perspective of powerless (versus powerful) others
}

Article in Cognition and Emotion · March 2021

DOl: 10.1080/02699931.2021.1902282

\section{CITATIONS}

6 authors, including:

Francois Quesque

French Institute of Health and Medical Research

25 PUBLICATIONS 322 CITATIONS

SEE PROFILE

E. Chabanat

Lyon Neuroscience Research Center

83 PUBLICATIONS 7,236 CITATIONS

SEE PROFILE

Some of the authors of this publication are also working on these related projects:

Hierarchy and Risk-Taking in the cockpit View project

Project post-doc 1 View project
CRNL

10 PUBLICATIONS 86 CITATIONS

SEE PROFILE

Yves Rossetti

Lyon Neuroscience Research Center

344 PUBLICATIONS 13,840 CITATIONS

SEE PROFILE 
This is a post-peer-review, pre-copyedit version of an article published in Cognition and Emotion. The final authenticated version is available online at: https://doi.org/10.1080/02699931.2021.1902282

\title{
Sympathy for the Underdog: People are inclined to adopt the emotional perspective of powerless (versus powerful) others
}

\begin{abstract}
Authors
François Quesque ${ }^{1,2^{*}}$, Alexandre Foncelle ${ }^{1,2^{*}}$, Elodie Barat ${ }^{1}$, Eric Chabanat ${ }^{1,2}$, Yves Rossetti ${ }^{1,2}$, and Jean-Baptiste Van der Henst ${ }^{1}$
\end{abstract}

\begin{abstract}
Affiliations
${ }^{1}$ Trajectoires Team, Centre de Recherche en Neurosciences de Lyon, Inserm UMR-S

1028,CNRS UMR 5292, Université Lyon 1, 95 Boulevard Pinel, Bron, France

2 Plateforme "Mouvement et Handicap" and Plateforme NeuroImmersion, Hôpital Henry-

Gabrielle, Hospices Civils de Lyon, 20 route de Vourles, Saint-Genis-Laval, France
\end{abstract}

* These authors contributed equally.

\section{Corresponding authors}

François Quesque : francois.quesque@gmail.com \& Jean-Baptiste Van der Henst :

vanderhenst@isc.cnrs.fr 


\begin{abstract}
Upon learning of the story of Cinderella, most people spontaneously adopt the emotional perspective of this helpless young woman rather than of her older sisters who oppress her. The present research examines whether this pattern reveals a general human tendency to empathize more with the emotions of individuals with low (versus high) power. Six experiments $(\mathrm{N}=878)$ examined how power influences the focus of people's emotional attributions. Participants were presented with situations in which one character exercised power over another one and had to resolve a referential ambiguity by considering the perspective of one or the other character. Results show that participants largely privileged the emotional states of the low-power character over those of the high-power character. This effect was observed with different types of stimuli (comics and video clips), with high- and low-power roles attributed to pairs of different genders (Experiments 1-4) or same gender (Experiments 5-6). Finally, the tendency persisted -though it was reduced- when participants adopted a less passive role with respect to the characters (Experiment 3) and when power occurred in a less despotic way (Experiment 6). Results are discussed with respect to social attention and sensitivity to fairness.
\end{abstract}

Keywords: Perspective-taking, Emotion, Power, Mentalizing, Referential ambiguity 


\section{Introduction}

Humans have developed sophisticated skills to identify and attribute emotions to their conspecifics. They infer emotions from very basic visual stimuli (Heider \& Simmel, 1944), they can perceive them unconsciously (Pegna, Khateb, Lazeyras, Seghier, 2005), and even newborns are able to discriminate emotional expressions (Farroni, Menon, Rigato \& Johnson, 2007). This ability allows people to take others' vantage point and adapt their behavior in face-to-face interactions. However, navigating the social environment not only requires the ability to take a second-person perspective, but also requires the ability to take a third-person perspective by passively observing the relations and interactions between two or more people.

Little is known about how people attend to others' emotions when they take such a third person perspective. An important issue is to identify the factors that lead to favor the perspective of one interactant over the other, regardless of the degree of familiarity or similarity between the observer and one of the agents. Symmetrical dyadic interactions, which involve reciprocity, should trivially lead to focus to the same extent on the two interactants, as each receives similar social input. However, a large part of human interactions are asymmetrical, which may lead to privilege more the perspective of one of the agents. The present research investigates how asymmetry in interactions influences the focus of people's emotional attribution. It concerns a very standard type of asymmetrical interactions, namely, situations where one individual exercises power over another, and therefore examines whether people are more likely to consider the emotions of the powerful or those of the powerless.

In power relationships, the dominant individual controls more resources and more valued outcomes than the subordinate, who is consequently more dependent on the dominant to achieve their goals. The ability to manipulate hierarchical notions such as power and status is fundamental for social life and emerges early in cognitive development. Preschool children and even infants are able to ascribe power from a variety of cues (e.g. body size, postures, 
age, resources) and asymmetric interactive exchanges (e.g. giving orders, setting norms, being imitated; Charafeddine et al. 2015; Gülgöz and Gelman 2017; Over and Carpenter 2015, Terrizzi et al., 2018, Thomsen et al., 2011; Pun et al., 2016). Moreover, social hierarchies are processed more easily and tend to be more liked than non-hierarchical information (Zitek \& Tiedens, 2012). For instance, people process faster a complementary pair composed of a dominant face and a submissive face, than equal pairs of two dominant or two submissive faces (Zitek \& Tiedens, 2012). This suggests that people clearly distinguish a dominant character from a subordinate one and even favor the differentiation between individuals in terms hierarchical status.

Social hierarchies influence emotion attribution. High-power individuals are expected to feel anger in negative situations, and pride in positive ones, while low-power individuals are expected to feel sadness and guilt in negative situations, and appreciation in positive ones (Tiedens, Ellsworth \& Mesquita, 2000). However, although people conceive power relationships according to different emotional perspectives, it is not clear which perspective they favor. In the present research, we investigate this issue and consider different factors that might induce participants to take one or the other perspective.

\section{Taking the perspective of the powerful}

Power holders are more salient and more influential in their environment. They tend to exhibit various physical devices (e.g. crowns, headgear and distinctive attire) and body postures (i.e. expanded and erect postures) that distinguish them from others (Hall, Coats \& LeBeau, 2005). Their influence in social interactions is also greater: they give more orders, they interrupt others' speech more often and they are more likely to set the topic of conversations (Hall et al. 2005). In addition, because powerful individuals can provide more reward and inflict more punishment, they are more likely to elicit vigilance of others. In order to maximize the benefits as well as minimize the costs of interactions with powerful 
individuals, investing cognitive resources in the monitoring of those individuals is thus an efficient strategy.

A wide range of results indicate that powerful and more generally high-status individuals are the target of others' cognitive resources. Observational studies on dominance hierarchies in primate societies and groups of preschool children show that the amount of attention allocated to individuals in a group correlates with their dominance status (Chance \& Larsen, 1976; Pellegrini, 2008; Abramovitch et al., 1976). Experimental research using eyetracking also reveals that human participants look more towards high-ranking contestants in competitions (Breton et al., 2018), individuals in high-status attire (Maner et al., 2008), and people who influence discussions more (Foulsham et al., 2011). They also look more towards the direction gazed by dominant faces than to the direction gazed by subordinate faces (Jones et al., 2010). Moreover, high-status male individuals tend to be remembered better than lower status individuals (Ratcliff et al., 2011). Research has also reported that monkeys are eager to obtain information about their high-status conspecifics, and can thus sacrifice a valuable resource to view a dominant peer (Deaner, Khera \& Platt, 2005). Taken together these results suggest that participants observing a power interaction may focus more on the emotions of the powerful than on those of the powerless. However, other factors may act in the opposite direction.

\section{Taking the perspective of the powerless}

First, there is evidence showing that people have positive feelings towards disadvantaged individuals, especially when they overcome their disadvantage. In popular culture and religions, the sensitivity to disadvantaged individuals who triumph from powerful individuals, or political institutions, is manifested through a variety of characters such as Cinderella, Rocky Balboa, David (vs. Goliath), Gandhi, and has been labelled the underdog effect (Kim et al., 2008). Experiments on the perception of competition and conflicts show 
that people tend to root for the underdogs and provide them more support than to top dogs (Kim et al., 2008; Vandello et al., 2007). For example, when asked to consider an upcoming sport competition between pairs of countries, people tend to show greater support for the underdogs than for the dominant countries (Vandello et al., 2007). Such support extends to psychological judgements about the underdogs, which are perceived as more attractive and more worthy than top dogs (Michniewicz \& Vandello, 2013). Accordingly, less powerful entities are also perceived as more moral than more powerful entities when engaged in intergroup hostilities (Vandello et al., 2011). Hence, the social preference towards individuals with less power could lead one to focus more on these individuals and their emotions.

Second, powerful and powerless differ in the emotions they experience. Because of the potentially unfair and displeasing behavior of high-power individuals, they are expected to feel socially disengaging emotions, like contempt, anger, or pride (Tiedens et al., 2001, Magee \& Smith, 2013; Kitayama et al. 2006). In contrast, low-power individuals are expected to experience more socially engaging emotions, like gratitude, guilt or embarrassment, which serve to maintain existing relationships or repair disrupted relationships (Tiedens et al., 2001; Magee \& Smith, 2013). Hence, since emotions of high-power individuals are socially more disengaging, they may elicit avoidance from a bystander.

Third, the emotions of the powerful may be more overlooked than those of the powerless. Power holders are associated with the motivation to act, to accomplish goals, and to obtain rewards (Fiske \& Neuberg, 1990; Keltner et al., 2003). The propensity to act and the associated behavioral manifestations could overshadow the emotions they experience in a social interaction less salient. In contrast, the emotions of subordinates are often direct consequences of the power-holders' behavior, which increases their salience. Moreover, powerful individuals are less dependent on others, which make them more socially distant (Magee \& Smith, 2013). Social distance of powerful individuals can create a barrier against 
the emotions of the people they interact with. This can be illustrated by a lower ability of high-power individuals to correctly assess the emotions of others (Galinsky et al., 2006; Gonzaga et al., 2008) but also by a lower level of emotion experienced in response to others' emotions (Van Kleef et al., 2008). People tend to perceive social distance in powerful individuals as they judge them as colder (Fiske \& Durante, 2019; Fragale, Overbeck \& Neale, 2011). It is thus possible that people are less inclined to attend the emotions of individuals exerting power on others.

\section{The present research and hypotheses}

The present research thus examines whether people, who observe a power interaction, privilege more the emotions of the powerful or privilege more the emotions of the powerless. On the one hand, the salience of powerful individuals and the vigilance they elicit in others could favor the former possibility. Conversely, sensitivity to disadvantaged individuals, combined with greater distance from the emotions of powerful people, could favor the latter possibility. We tested these two hypotheses in a paradigm where participants observed a power asymmetry between two characters, and then had to resolve a referential ambiguity (e.g. Niewland \& Van Berkum, 2008) by selecting either the emotional perspective of the high-power character or that of the low-power character. In Experiments 1 and 2, participants had to adopt a bystander position while in Experiment 3, they had to take a less passive role towards the characters. Experiment 4 tested whether the results obtained for spontaneous emotional attribution would extend to the perceptual domain, by resolving a visuospatial ambiguity, leading to take either visuospatial perspective of the powerful or the one of the powerless. Finally, Experiments 5 and 6 respectively used a more ecological material (video clips with professional actors) and involved another expression of power.

\section{Experiment 1}


In Experiment 1, participants observed a power asymmetry and had to respond to the following question: "According to you, at this precise moment, what does this person feel?" This allowed us to test whether people privileged more the emotions the high-power character or privileged more the emotions of the low-power character. The salience of powerful individuals would favor the former possibility while sensitivity to disadvantaged individuals would favor the latter. When conducting this first experiment, we had no strong preference for one of these two alternatives.

\section{Method}

\section{Participants}

One-hundred and twenty-one young adults (mean age $=26.1,82$ females) participated in Experiment 1, which was conducted online. They were current or former students from the university of Lille (France) and were invited to participate in an online announcement. Sensitivity power analysis $(1-\beta=.80, \alpha=.05$, one-tailed) allowed us to identify a Cramer's V effect size of 0.25 with a probability of .80 (Faul, Erdfelder, Lang \& Buchner, 2007).

\section{Materials}

The interaction presented to the participants consisted in a cartoon story made of 13 comic strips (all materials and data are available at: https://osf.io/tf68r/) in which a male character exercises power over a female character. Different gender characters were used to better identify to whom the participants referred in their responses since they could use different pronouns. On several occasions, the high-power character ordered the low-power character to bring him an item. The low-power character did her best to comply with the requests of the high-power character, but the latter was never satisfied (See Figure 1 for the detailed script of the verbal interaction). On the last box, the two characters displayed different emotional states in line with their power status (Tiedens et al., 2000). The highpower character verbally expressed dissatisfaction and showed facial and body postures 
associated with anger and dominance (Hall et al., 2005), namely, lowered eyebrows, erect posture and arms raised (see Figure 2A). Conversely, the low-power character displayed features associated with distress and subordination (Hall et al., 2005), namely, raised eyebrows, bowing posture and arms close to the body (see Figure 2A).

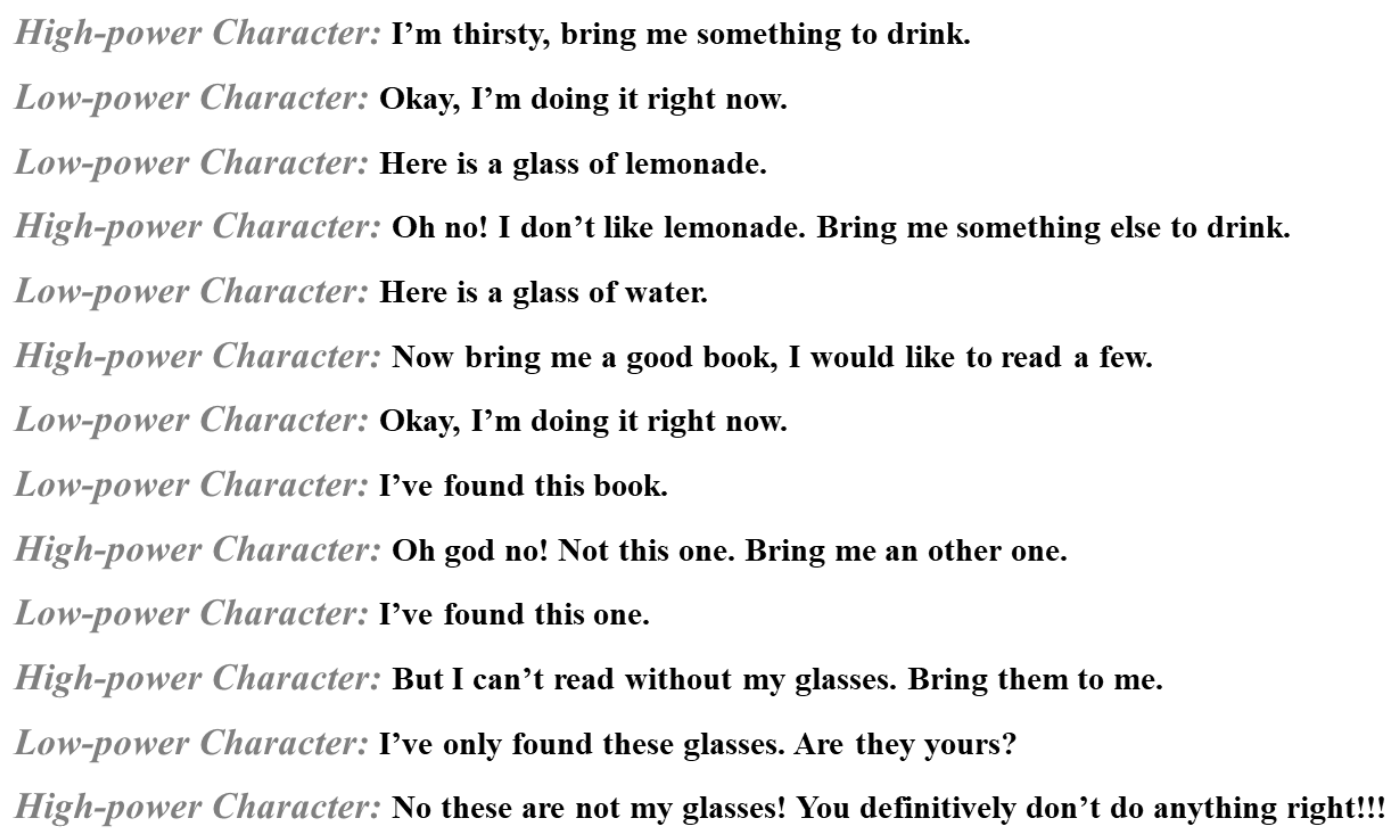

Figure 1. The detailed script of the verbal interaction presented in the comic strips of Experiments 1-4 and the video clip of Experiment 5.

\section{Procedure}

Participants watched the comic-strip according to a self-paced procedure. Although participants were given as much time as necessary to watch each individual strip, once they had moved on to the next strip they could not review the previous strips. Next, they had to answer the following ambiguous question: "According to you, at this precise moment, what does this person feel?". The ambiguity lies in that the question referred to a single individual whereas two individuals were interacting. In line with earlier studies (Hanna et al., 2003; 2004; see also Boroditsky \& Ramscar, 2002; Hauser, Carter \& Meier, 2009), participants had to disambiguate the reference by adopting either the emotional perspective of the high-power character, or that of the low-power character. Although participants were invited to consider the emotions of a single character, with the use of a singular demonstrative (i.e. "this 
character"), participants were not forbidden to take the perspective of both characters. This could occur if participants did not want to privilege one or the other perspective. No other questions regarding the comic strip were asked to participants. After they completed the task, they were asked for their age and gender.

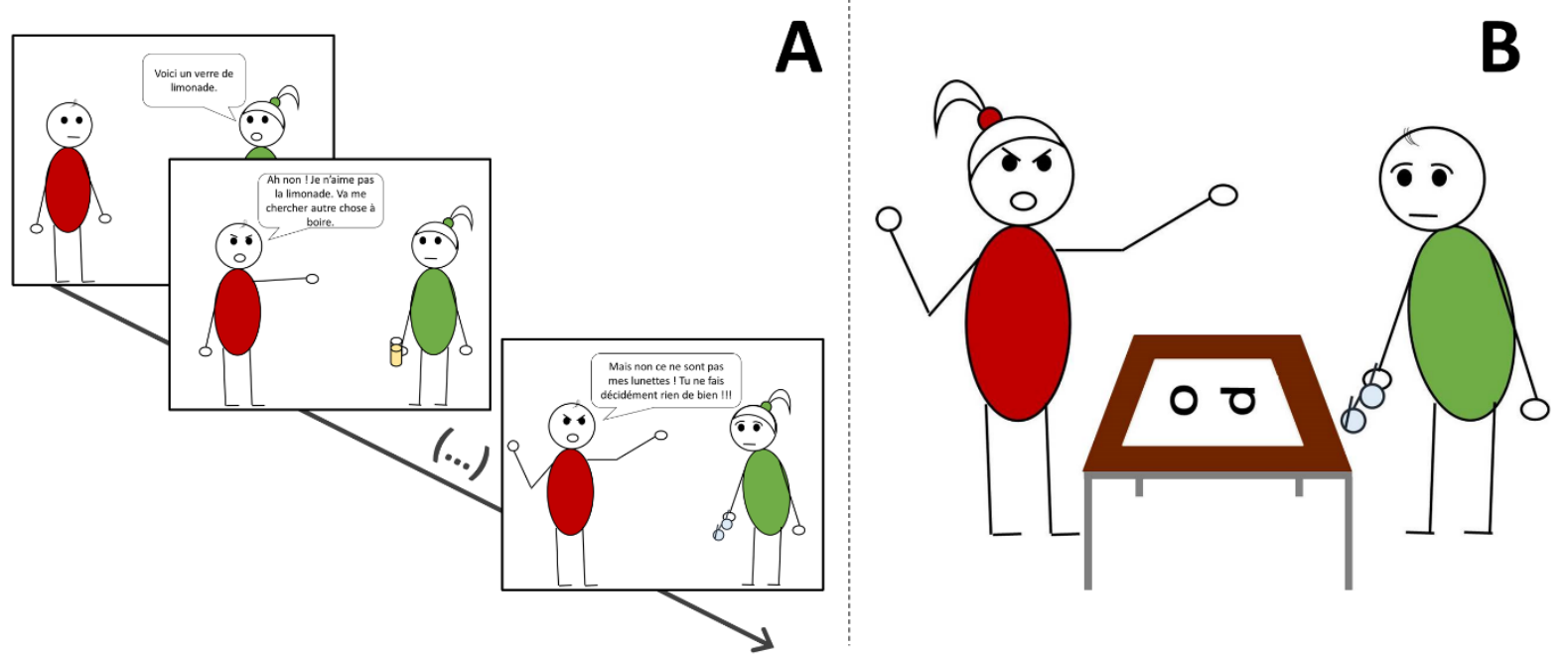

Figure 2. (A) Illustration of the type of comic strip used in the experiments 1-4 and 6 (samples displayed here originated from Experiment 1), in which a high-power character ordered a low-power character to bring different items, while never being satisfied (see supplementary materials for the full, 13 boxes, comic strips related to each experiment). (B) Illustration of the stimulus used for the spontaneous visuospatial perspective-taking judgement in Experiment 4.

\section{Results and discussion}

Two naïve judges individually categorized (inter-judge reliability index, Cohen's Kappa $=0.95)$ the responses as "high-power perspective" (e.g. "angry", "irritated"), "lowpower perspective" (e.g. "sad", "disappointed"), or "both perspectives" (e.g. "the green one is sad and the red one is angry") according to the type of emotions described or the grammatical gender of the character to which the participants referred to. When one judge could not confidently categorize the perspective taken, the response was categorized as "ambiguous" (e.g. "tired", "misunderstanding") and excluded from analysis (12 participants).

Participants took significantly more the perspective of the low-power character than the perspective of the high-power character $\left(64.2 \%\right.$ vs. $7.3 \%, \chi^{2}(1)=49.028, p<.001, V=0.79$, 
see Figure 3 ) and $28.8 \%$ of participants took the perspective of both characters. The perspectives taken did not significantly differ between female and male participants (Fisher's Exact Test $\mathrm{p}=.149$, females: low-power character $70.2 \%$, high-power character $6.8 \%$, double 23\%; males: low-power character 51.5\%, high-power character $8.5 \%$, double $40 \%$ ). The results support the view that participants focused more on the emotions of the low-power character than those on the high-power character. However, one aspect of the methodology that might have led to focus more on the subordinate agent regardless of the power interaction is grammatical gender. In the question asked to participants, the noun "person" ("personne" in French) is of feminine grammatical gender. This might have led participants to think that it referred more to the female than to the male character. Consequently, Experiment 2 addressed this issue by reversing the gender of the high- and low-power characters.

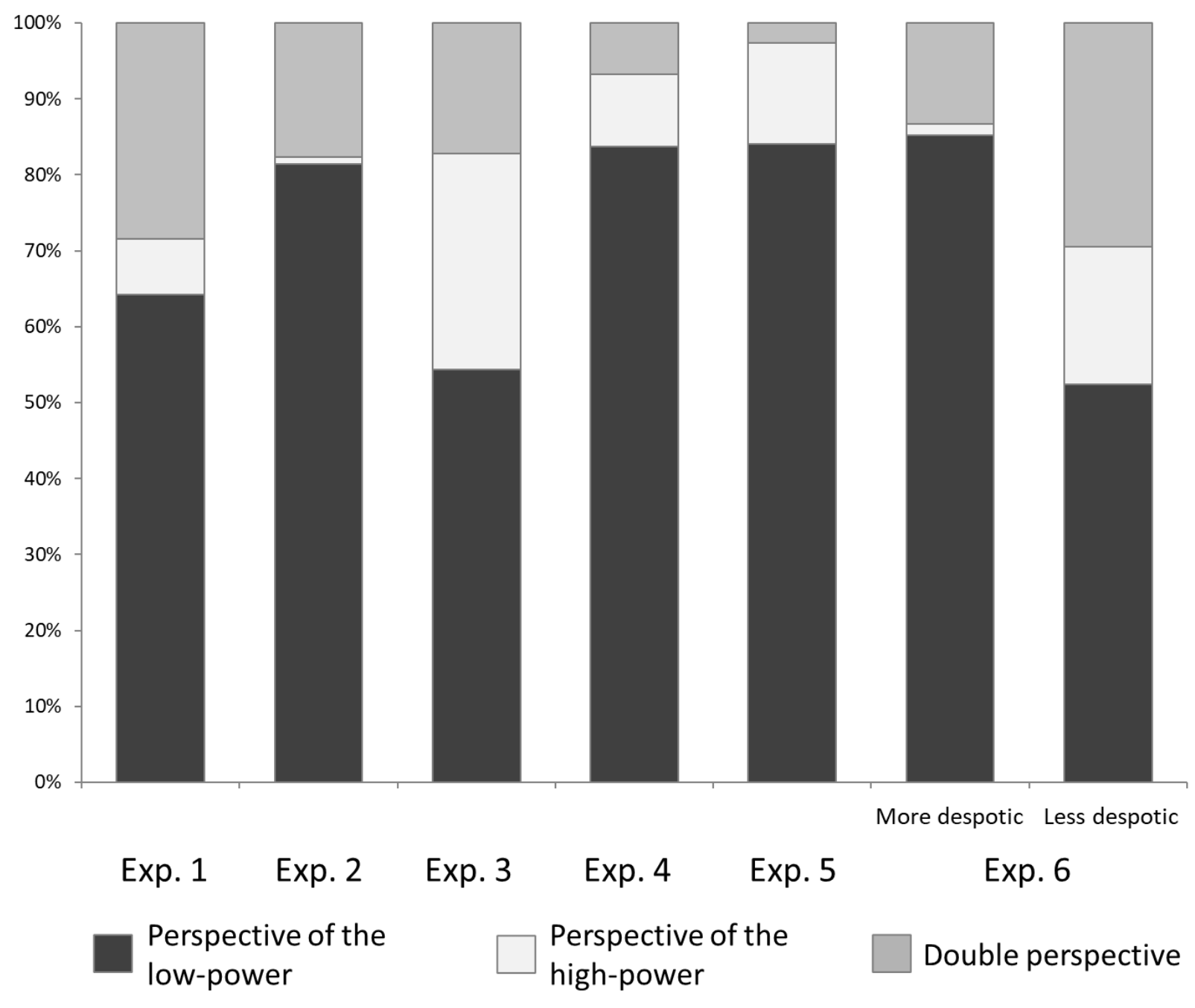


Figure 3. Proportion of responses referring to the low-power character (black), the high-power character (light grey), or both (grey) in Experiment 1-6.

\section{Experiment 2}

In Experiment 2, participants saw the same power interaction as in Experiment 1, but this time the high-power character was a female, and the low-power character was a male. In accordance with the results of Experiment1, we expected participants to focus more on the emotions of the low-power character than on those of the high-power character.

\section{Method}

\section{Participants}

One-hundred and twenty-eight young adults (mean age $=21.8,97$ females) participated in Experiment 2, which was conducted online. They were students from the university of Lyon (France) contacted by an online announcement and did not participate in Experiment 1 . Sensitivity power analysis $(1-\beta=.80, \alpha=.05$, one-tailed $)$ allows us to identify a Cramer's V effect size of 0.24 with a probability of .80 .

\section{Materials and procedure}

The materials and the procedure were identical to that of Experiment 1 except that the female character was dominant, and the male character was subordinate. Moreover, right/left locations of the characters were counterbalanced across participants.

\section{Results and discussion}

Responses were categorized in the same way as in Experiment 1 (inter-judge reliability index, Cohen's Kappa $=0.95)$. Fifteen participants provided ambiguous responses that were excluded from the analysis. As in Experiment 1, participants took the perspective of the subordinate to a greater extent than that of the dominant ( $81.4 \%$ vs. $0.9 \%$, Fisher's Exact Test $\mathrm{p}<.001, \mathrm{~V}=0.98)$ and $17.7 \%$ of participants considered the perspectives of both characters. The perspectives taken did not significantly differ between female and male 
participants (Fisher's Exact Test $p=.31$, females: subordinate 83\%, dominant 0\%, double $17 \%$, males: subordinate $79.2 \%$, dominant $4.2 \%$, double $16.6 \%$ ) and nor of the left-right position of the characters (Fisher's Exact Test $\mathrm{p}=.99$ ). By replicating the effect found in Experiment 1, Experiment 2 confirms that people are more inclined to consider the emotional state of the subordinate rather than the one of the dominant. It demonstrates that this effect cannot be accounted for by an influence of the grammatical gender of the noun "person" used in the question. Interestingly, a cross-experiment comparison even shows that more participants took the perspective of the subordinate in Experiment 2 than in Experiment 1 $\left(81.4 \%\right.$ vs. $\left.64.2 \% \chi^{2}(1)=11.1, \mathrm{p}=.001, \mathrm{~V}=0.16\right)$. Although this difference was unexpected, it could be explained by the inversion of a gender hierarchy in Experiment 2, which was incongruent with societal norms (i.e. male subordinate and female dominant) and might have attracted attention to the subordinate even more than in Experiment 1 (Ridgeway, 2001; Wagner \& Berger, 1997).

Although in Experiments 1 and 2 participants were unrelated to any of the two characters, they were placed in a passive role due to their bystander position. This might have contributed to their focusing on the most passive character in the interaction, that is, on the low-power character. In the next experiment, we aimed to manipulate the inclination towards the subordinate's emotional perspective by modifying the participant's position. In many social situations, people move from the role of a bystander to a more active role that leads them to interact directly with the individuals they initially observed. This shift may influence the consideration of others' mental states, because interacting with socially distinct agents can result in very different outcomes. Given that a high-power individual is more likely to influence others than a low-power one, it might be more critical to consider the consequences of interacting with the former than with the latter, and thus to focus more on the former's emotions. 


\section{Experiment 3}

In Experiment 3, participants still had to describe the feelings of a character, but also had to imagine that they would interact with him or her. We thus aimed to examine whether the tendency to take the perspective of the passive character remains when participants adopt a less passive role. Specifically, we postulated that the adoption of a less passive role would reduce the tendency to focus more on the low-power character's emotions.

\section{Method}

\section{Participants}

One-hundred and thirty-three young adults (mean age $=27.1,82$ females) participated in Experiment 3, which was conducted online. They were current or former students from the university of Lyon (France) invited to participate by e-mail and did not participate to the previous experiments. Sensitivity power analysis $(1-\beta=.80, \alpha=.05$, one-tailed $)$ allows us to identify a Cramer's V effect size of 0.24 with a probability of .80 .

\section{Material and procedure}

The material was identical to that of Experiment 2 but the procedure differed in that the participants had to project themselves into the situation when answering the ambiguous question. Hence, after reading the comic-strip, participants received the following instruction: "Imagine now that you will have to interact with that person. According to you, at this precise moment, what does this person feel?". To avoid participants to be influenced by the nature of the interaction, we did not specify its content. A negative interaction between one character and the participants (e.g. a conflict) could incite them to take the perspective of the powerful character as this character also behaves in a more negative way towards the powerless individual, while a positive interaction, could incite participants to take the perspective of the low-power individual, who behaves positively towards the dominant. Participants simply had to imagine that they would interact with one of the characters. 


\section{Results and discussion}

Responses were scored as in Experiment 1 (inter-judge reliability index, Cohen's Kappa $=0.94)$. Seventeen participants provided an ambiguous answer and were excluded from analysis. As in Experiments 1 and 2, more participants took the perspective of the lowpower character than that of the high-power character $\left(54.3\right.$ vs. $28.4 \% ; \chi^{2}(1)=9.38, p=.002$, $\mathrm{V}=0.31$ ). However, the rate of participants who took the perspective of the high-power character was much higher than in Experiment 2 (28.4\% vs. $0.9 \%$, Fisher's Exact Test $\mathrm{p}<$ $.001)$. Finally, $17.2 \%$ of participants provided answers from both perspectives. The perspectives taken did not significantly differ between female and male participants (Fisher's Exact Test $\mathrm{p}=.183$, females: subordinate $49.3 \%$, dominant $28.8 \%$, double $21.9 \%$, males: subordinate $62.8 \%$, dominant $27.9 \%$, double $9.3 \%$ ). While replicating the inclination to privilege the emotional perspective of the subordinate, the present results also suggest that attending to others' emotions partially depend on the possibility to interact with them.

Experiments 1-3 examined perspective-taking through the lens of emotions. However, the consideration of others' vantage point also involves cognitive (e.g. their beliefs or their knowledge) and perceptual (e.g. what they visually perceive from their spatial position) aspects. Several studies indicate that different types of perspective-taking judgements can influence each other, which suggests that this ability works similarly across domains. For instance, participants who are good at ascribing emotions to others tend to also be better at visuospatial perspective-taking (Erle \& Topolinski, 2015; Mattan, Rotshtein \& Quinn, 2016). Moreover, when participants are induced to take another's visuospatial perspective, they also tend to attribute more conceptual mental states to others (Erle \& Topolinski, 2017).

Conversely, some studies reported no cross-domain influences of other-oriented skills. For instance, a study found that feeling compassion for someone does not result in taking more their visuospatial perspective (Quesque, Chabanat \& Rossetti, 2018). Moreover, 
interactions do not affect all protagonists' mental states in the same way. While participants may easily conceive that power asymmetries alter emotional states of social agents (Tiedens et al., 2002), it is not clear whether they could envisage that power differentially affects the visuospatial perception of those agents. A lack of differentiation of mental states in the spatial domain might result in attributing the same weight to each agent's perspective. In Experiment 4, we therefore investigated whether focusing on the emotional perspective of a low-power character also leads to take more their visuospatial perspective.

\section{Experiment 4}

In Experiment 4, we tested whether participants' tendency to favor the perspective of the low-power individual extend to the visuospatial domain. Participants first had to answer the same ambiguous question as in Experiments 1 and 2, so as to anchor them on the emotions of the low-power individual, and then had to answer a spatially ambiguous question. In accordance with the findings of Quesque et al. (2018), we expected a relative independency between the emotional and the perceptual domains.

\section{Method}

\section{Participants}

One-hundred and fourteen young adults (mean age $=22,67$ females) participated in Experiment 4, which was conducted online. They were students from the university of Lyon. They were contacted by email and did not participate to the previous experiments. Sensitivity power analysis $(1-\beta=.80, \alpha=.05$, one-tailed $)$ allows us to identify a Cramer's V effect size of 0.26 with a probability of .80 .

\section{Materials and procedure}

First, participants received the same power asymmetry and the same ambiguous question as in Experiment 2. We could thus to analyze the same dependent measure (i.e. the 
focus on the high-power or low-power emotional perspective) as in this experiment. Then, they were presented with another picture including the same two characters and two letters (see Figure 2B). They were then asked another ambiguous question regarding letters shown in the picture: "Letters are displayed on the table. What are these letters?" Letters could be read differently depending on the perspective adopted. Such "letter-identification" task has been used to probe human's tendency to spontaneously take another perspective (Arnold et al., 2016; 2019; Quesque et al., 2018). Taking the spatial perspective of the subordinate should lead to read the letters as "o" and "d", while taking the perspective of the dominant should lead to read the letters as "p" and "o".

\section{Results and discussion}

Responses were scored as in Experiment 1 (inter-judge reliability index, Cohen's Kappa $=0.79$ ). The perspective endorsed was ambiguous for 11 responses, which were excluded from analysis. As in earlier experiments, there were more participants who took the emotional perspective of the subordinate agent (83.5\%) than the one of the dominant agent $\left(9.7 \%, \chi^{2}(1)=59.21, p<.001, V=0.79\right)$. Additionally, $6.8 \%$ of participants responded using the perspectives of both agents. The perspectives taken did not significantly differ between female and male participants concerning the target of the perspective-taking judgement (Fisher's Exact Test $\mathrm{p}=.51$, females' proportion: subordinate $87.1 \%$, dominant $8.1 \%$, double $4.8 \%$, males' proportion: subordinate $78 \%$, dominant $12.2 \%$, double $9.8 \%$ ).

Concerning the visuospatial subsequent task, a "o-d" answer indicated the subordinate's perspective, a "p-o" answer indicated the dominant's perspective and " $\mathrm{d}-\mathrm{p}-\mathrm{o}$ " answer indicated a double perspective. Participants who initially took the emotional perspective of the low-power individual did not then take more their spatial perspective than that of the high-power individual $\left(30.5\right.$ vs. $\left.37.8 \%, \chi^{2}(1)=0.64, p=.42, V=0.10\right)$. In addition, $31.7 \%$ took both perspectives. In order to get more conclusive information regarding the latter 
comparison, we computed the Bayes factors with the version 0.9.8 of the BayesFactor package for R (Rouder, Speckman, Sun, Morey \& Iverson, 2009). As recommend by Schönbrodt et al. (2017), in absence of previous knowledge, we used the less informative JZS prior with $r=1$ to scale the Cauchy distribution. Consistently, the Bayes factor obtained for the comparison supports the null hypothesis $\left(\mathrm{BF}_{01}=4.81\right.$, which is however relatively anecdotal according to Jeffreys, 1961). Finally, among the 10 participants who initially took the emotional perspective of the dominant, 5 also took her visuospatial perspective. Hence, as in previous experiments, participants focused more on the emotional perspective of the lowpower agent, but this tendency did not extend to the visuospatial domain. This independence between the emotional and visuospatial social inferences is congruent with previous works (Quesque et al., 2018) and could reflect the existence of independent cognitive processes supporting different kinds of social inferences (Shamay-Tsoory \& Aharon-Peretz, 2007; Shamay-Tsoory, Aharon-Peretz \& Perry, 2009).

We now want to discuss one aspect of the stimuli used to implement the power interaction in the four experiments. Although the tendency to focus on the subordinate's emotions was always observed, the four experiments all relied on a single stimulus, namely a comic strip, which might not be representative of all possible stimuli (see Judd, Westfall \& Kenny, 2012; 2014; Westfall, Judd \& Kenny, 2015). Therefore, it cannot be excluded that the observed effect was attributable to the specificity of our stimulus. In popular culture, comics and cartoons often depict characters with an imbalance of power (e.g. Tom and Jerry, Tweety and Sylvester, Mario and Bowser), and in most cases the viewer is invited to identify more with the low-power character than with the high-power character. To avoid any reminiscence of such an invitation, Experiment 5 was no longer based on a comic book paradigm.

\section{Experiment 5}


As in previous experiments, participants saw a power interaction between two characters. However, this time, the interaction was filmed and played by real humans. This allowed us to examine whether the results obtained with cartoon characters could also be found in more real-life situations. In accordance with the results of Experiments 1-4, we expected participants to still focus more on the emotions of the low-power character than on those of the high-power character.

\section{Method}

\section{Participants}

One-hundred and thirty-two young adults (mean age $=21.7,117$ females) participated in Experiment 5, which was conducted online. They were medical students, invited to participate by an announcement made by the end of a lesson and did not participate in any other experiments linked to the present manuscript. Sensitivity power analysis $(1-\beta=.80$, $\alpha=.05$, one-tailed) allows us to identify a Cramer's V effect size of 0.24 with a probability of .80 .

\section{Materials}


We used short (1 minute) movies in which two male actors (one professional and one semi-professional) played either the role of dominant character or that subordinate character (see Figure 4. for an illustration). The spatial location (left vs. right) of the actors and the roles they were attributed (dominant vs. subordinate) were counterbalanced, resulting in 4 short movies. The scenario and dialogues were identical to those of the comic strips used in Experiments 1-4 (see Figure 1 for the script). Each participant viewed a single movie.

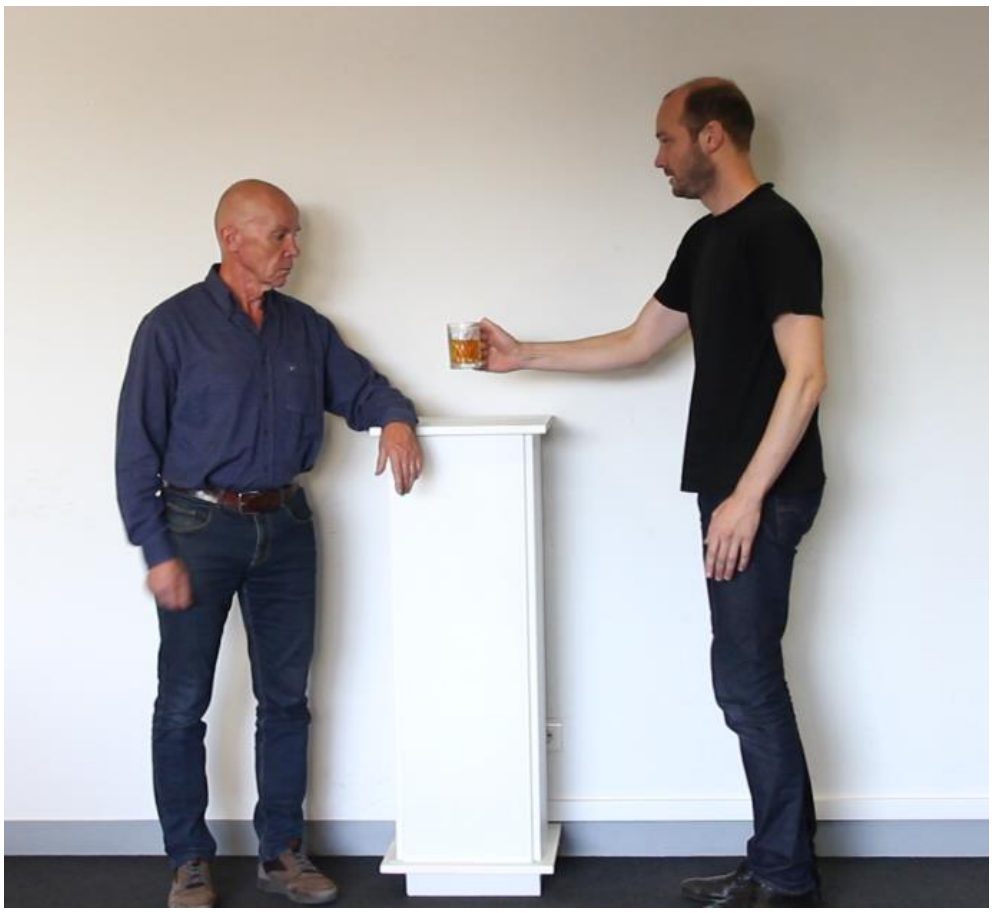

Figure 4. Illustration of the short film used in Experiment 5 and adapted from the comic strip used in Experiments 1-4.

\section{Procedure}

Participants watched the movie and then had to answer the same question as in earlier experiments: "According to you, at this precise moment, what does this person feel?".

\section{Results and discussion}

Responses were scored using the same criteria as in Experiment 1 (inter-judge reliability index, Cohen's Kappa $=0.93$ ). The perspective endorsed was ambiguous for 19 responses, which were excluded from analysis. In accordance with the results obtained in experiments 1-4, a vast majority of participants took the emotional perspective of the 
subordinate character $(84.1 \%)$ rather than the one of the dominant character $(13.3 \%$, $\left.\chi^{2}(1)=58.18, p<.001, V=0.72\right)$. Also, we found no significant difference between female and male participants (Fisher's Exact Test $\mathrm{p}=.80$, females' proportion: subordinate $82.8 \%$, dominant $14.2 \%$, double $3 \%$, males' proportion: subordinate $92.9 \%$, dominant $7.1 \%$, double 9.3\%). Additionally, $2.7 \%$ of participants responded using the perspectives of both agents. Experiment 5 replicates the preference for the subordinate's emotions in proportions similar to what was observed in earlier experiments, while relying on more ecologically valid stimuli. Moreover, participants were here confronted to a social interaction implying two actors of the same gender (i.e. male).

We now want to discuss two aspects of the interaction presented in the experiments so far that might have favored the emotional perspective of the subordinate. First, in the current scenario, the high-power character repeatedly told the low-power character that he was unable to comply with their requests. However, the low-power character had no way of realizing that their actions could not satisfy the high-power character, since they were always compatible with those requests. Consequently, the manifestation of the high-power character's dissatisfaction may seem quite inappropriate and could give the impression that the dominant was particularly bossy and treated the low-power character far too unfairly. This could have caused participants to focus more on what he was going through than they would have done so with a less despotic expression of power. In order to neutralize this potential effect, Experiment 6 introduced a situation where the high-power individual no longer expressed inappropriate negative judgments regarding the way the low-power character was trying to comply.

Second, another issue concerns turn taking. Since the high-power character is the last to speak, participants might focus more on what the addressee feels about the high-power individual's speech, especially if that speech voices a negative judgment made on the low- 
power individual. In addition, participants may focus more on the low-power individual because they may expect that it is her turn to speak. In Experiment 6, it is the low-power character who was the last to speak. Moreover, to limit the influence of turn taking, the two characters were shown with symmetrical thought bubbles reflecting their reflection on what previously happened.

\section{Experiment 6}

In Experiment 6, we compared the emotional perspective taking in a relatively despotic power situation, similar to previous experiments, to a less despotic power situation. We expected that the tendency to focus more on the emotions of the low-power character than on those of the high-power character would be more pronounced in the more despotic condition than in the less despotic condition. In addition, we also sought to examine the influence of participants' perceptions of their own power on their emotional attributions.

\section{Method}

\section{Participants}

Two-hundred and fifty young adults (mean age $=22.8,176$ females) participated in Experiment 6, which was conducted online. They were medical students, invited to participate by an announcement made by the end of a lesson and did not participate in any other experiments linked to the present manuscript. Considering the between-subject design that was used in Experiment 6, sensitivity power analysis $(1-\beta=.80, \alpha=.05$, one-tailed $)$ allowed us to identify a Cramer's V effect size of 0.20 with a probability of .80 .

\section{Materials and design}

Since the comic and the short film produced similar results, we returned to the former comic-strip paradigm for the sake of simplicity. A power interaction between two characters 
with no hair (to limit gender differentiation between characters), one blue and one yellow, was shown to the participants in two different conditions. In the "more despotic" condition, the high-power character gave the same five orders to the low-power character as in Experiments 1-5, that is, three different orders and two reformulated orders following the expression of his discontent. In the "less despotic" condition, the scenario and the dialogues were modified so that the high-power character no longer expressed inappropriate negative judgments regarding the attempts of the low-power character to meet his requests. The highpower character gave five different orders to the low-power character and only the last request was not met: the high-power character asked for his red book, but the low-power character brought him a green book. In contrast with the "more despotic" condition, where only the high-power character could understand that his requests were not met, in the "less despotic" condition both characters realized that the final request was not met. This was represented by two thought bubbles. For the low-power character the bubble was "Damn, this is not his red book" ("Mince, ce n'est pas son livre rouge"), for the high-power character it was "But, this is not my red book" ("Mais, ce n'est pas mon livre rouge”). The comic strip and the dialogues used in the two conditions are available at: https://osf.io/tf68r/. Participants were randomly assigned to one of the two experimental conditions. In both conditions, the spatial location (left vs. right) and the role attributed to each character (high-power vs. low-power) were counterbalanced across participants.

\section{Procedure}

As for earlier experiments, participants watched the comic-strip according to a selfpaced procedure. Next, they had to answer the following ambiguous question: "According to you, at this precise moment, what does this person feel?". Once they had validated their response, a subsequent question was displayed. Participants were explicitly asked to specify to which character they were referring to in the previous question (Three options were 
presented: the subordinate, the dominant, both characters). This additional question offered a supplementary validation of the procedure's internal validity, comforting the overall high inter-judge reliability in Experiments 1-5. Finally, participants had to answer a short questionnaire (15 items, see Table 1) adapted by Freeman, Rule, Adams \& Ambady, (2009) from Goldberg et al.'s (2006) International Personality Item Pool. This questionnaire indexes behavioral tendencies towards dominance versus subordination. Participants had to assess each questionnaire item on a five-point scale. (higher scores reflecting dominance tendencies, maximum score $=75$ ). Logistic regression from individual scores at the questionnaire allowed to test whether the tendency to focus on the low-power character's emotion is linked to a greater experience in the subordinate position, which could be the case of our relatively young sample.

Table 1. Questionnaire items indexing behavioral tendencies towards dominance versus subordination.

\begin{tabular}{ccc} 
Items & Statement & Category \\
\hline $\mathbf{1}$ & I try to surpass others' accomplishments. & I want to control the conversation. \\
$\mathbf{3}$ & I am not afraid of providing criticism. & Dominant statement \\
$\mathbf{4}$ & I challenge others' points of view. \\
$\mathbf{7}$ & I lay down the law to others. \\
$\mathbf{9}$ & I put people under pressure. \\
$\mathbf{1 0}$ & I impose my will on others. \\
$\mathbf{1 1}$ & I try to lead others. \\
$\mathbf{1 2}$ & I take control of things. \\
$\mathbf{1 3}$ & I am the first to act. \\
\hline $\mathbf{5}$ & I express myself easily.
\end{tabular}


6

14

15
I let others make the decisions

I am not highly motivated to succeed.

I can't come up with new ideas.
Subordinate statement

(reversed score)

\section{Results and Discussion}

As for the earlier experiments, participants took more the emotional perspective of the low-power character than that of the high-power character (the range of emotions ascribed and their respective frequencies are available at: https://osf.io/tf68r/). This was observed in the "more despotic" condition $\left(85.2 \%\right.$ vs $\left.1.6 \%, \chi^{2}(1)=103.14, \mathrm{p}<.001, \mathrm{~V}=0.96\right)$ but also in the "less despotic" condition $\left(52.5 \%\right.$ vs $\left.18 \%, \chi^{2}(1)=20.51, \mathrm{p}<.001, \mathrm{~V}=0.49\right)$. The proportions of participants who took the perspective of both characters were respectively of $13.3 \%$ and 29.5\%. Despite the tendency to focus on the low-power character's emotions (see Figure 3), we observed a significant deviation of the responses from the flat distribution $\left(\chi^{2}(2)=35.06, p\right.$ $<.001, \mathrm{~V}=0.30)$, revealing distribution differences between the two conditions. Two-by-two comparisons revealed that participants significantly took more often the perspective of the low-power character in the "more despotic" condition than in the "less despotic" condition (85.2\% vs. $52.5 \%, \mathrm{Z}=5.6, \mathrm{p}<.001 ; \mathrm{BF}_{10}=184851$, which very strongly supports the alternative hypothesis, Jeffreys, 1961). As Experiments 1-5, we found no significant difference between female and male participants. There was no effect of participants' gender in the "less despotic" condition (Fisher's Exact Test $\mathrm{p}=.12$, females' proportion: subordinate $86.8 \%$, dominant $0 \%$, double $13.2 \%$, males' proportion: subordinate $81.1 \%$, dominant $5.4 \%$, double $13.5 \%$ ) as well as in the "more despotic" condition (Fisher's Exact Test $p=.123$, females' proportion: subordinate $52.9 \%$, dominant $14.1 \%$, double $33 \%$, males' proportion: subordinate $52.8 \%$, dominant $2.8 \%$, double $19.4 \%$ ). Finally, scores to the "dominance versus subordination" questionnaire were well distributed around the mid-value (mean=42.2, $\mathrm{sd}=$ 9.14 , range $=20-68$, indicating that participants usually did not seem to represent themselves in a subordinate position more than in a dominant position. In order to test if participants' 
experienced dominance influenced their tendency to consider the low-power character's emotional perspective in experiment 6 , we used a logistic regression model of the probability of taking the subordinate's perspective with participants' scores at the questionnaire as explaining variable. Interestingly, the "dominance versus subordination" score did not significantly contribute to the model $($ Coef $=0.024, Z=1.58, p=0.11)$, suggesting again a relative independence between participants' characteristics and the effects reported through this manuscript.

\section{General Discussion}

The present research explored which emotional perspective people take when they are observing an interaction between a more powerful person and a less powerful person. Across six experiments, we have examined how power influences the focus of people's spontaneous emotional attribution. Participants were presented with situations (a 13 boxes comic strip or a 1-minute movie clip) in which a character exercised power over another one. The results indicated that participants largely privileged the emotional states of the low-power character over those of the high-power character. This effect was observed i) with different types of stimuli (comics and film, Experiment 5), ii) with high- and low-power roles attributed to different genders (Experiments 1 and 2), iii) with a more or less despotic expression of power (Experiment 6), and iv) with participants adopting a less passive role (Experiment 3).

Moreover, this effect was independent of participants' gender (Experiment 1-6) and selfreported dominance (Experiment 6).

The greater social attention allocated to high-power individuals, which has been often reported (Chance \& Larsen, 1976; Pelligini, 2008; Abramovitch et al., 1976), does not automatically lead to a greater consideration of their emotions. This suggests that when monitoring high-power individuals, people pay attention to other internal (as well as external) features than to their emotions. In particular, they might focus more on their intentions or 
motivations in order to anticipate and adapt to their next action. The reasons for the greater consideration of the powerless emotions remains to be discussed and explored further, but the results of the present research provide some possible clues.

A first possible mechanism is the human sensitivity to fairness and inequality, which emerges early and which may lead to favor the underdog. In the specific case of dominance interactions, children around the age of 5 begin to favor the subordinate over the dominant in resource distribution tasks (Charefeddine et al., 2016; Enright et al., 2020). Moreover, infants show a preference for subordinate individuals over dominant individuals who impose themselves by force (Thomas et al., 2018). Among adults, fairness considerations are put forward to account for the underdog effect (Kim et al., 2008; Vandello, et al., 2007), which shows a greater support to disadvantaged individuals and groups in competition (Kim et al., 2008. Vandello, et al., 2007). This inclination could orient the representation of emotions on those individuals, and this focus could vary according to the degree of fairness. The results of Experiment 6 are in line with this possibility as when the situation was more unfair, that is, when the high-power character was more despotic, participants took more the perspective of the low-power character. Moreover, in the present experiments, the low-power characters displayed a strong sense of commitment and effort (systematically trying to satisfy the highpower's desires), which has been found to prompt support for the underdog (Vandello et al., 2007). It is also important to underline that our study differs in two aspects from previous work on the underdog effect. First, our tasks did not concern conflict nor competition but only focused on power situations. Second, and more importantly, the dependent variable we examine is not framed in terms of support or sympathy towards the powerless, but rather in terms of emotional perspective taken.

A second factor that seems to modulate the emotions considered in power interactions, is the degree of participants' implication. In Experiment 3, where participants were less 
passive observers, they were more likely to take into consideration the emotions of the highpower individual. In the experimental procedure, the possibility for the participants to interact with one of the characters was presented before the test question. This probably led to a greater attention allocated to the potential consequences of interacting with high-power individuals. Investing cognitive resources in the monitoring of those individuals could have been judged as less necessary when people were not directly affected by social interactions. Interestingly, this echoes results showing that self-relevance can attenuate the support given to disadvantaged individuals. For instance, Kim et al. (2008) observed that participants are less likely to support the underdog when the competition between the underdog has direct consequences for them. However, even if the proportion of participants who considered the perspective of the high-power individual increased in Experiment 3 as compared to Experiment 2, it remains striking that most participants still favored the emotions of the subordinate.

Third, participants might have thought that experiencing power reduces emotional sensitivity. High-power individuals show reduced emotional contagion (Van Kleef et al., 2008) and are perceived as colder than low-power individuals (Fiske \& Durante, 2019; Fragale, Overbeck \& Neale, 2011). Moreover, even when displaying feelings, the emotions they are attributed is of limited range (Brescoll \& Uhlmann, 2008; Hareli, David \& Hess, 2013; Tiedens et al., 2000). Given that those individuals are associated with goal achievements, rewards and actions, people might focus more on the driving force of those features, that is on motivation or affordances, rather than on emotions. However, the present experiments did not allow to disentangle between the sensitivity to fairness and the lower attribution of emotions to the high-power individual. To better isolate this latter factor, future research could ask participants to assess the intensity of the emotions felt by each interactant. 
Moreover, it is also possible that the two factors are interrelated, and that participants attribute even more emotional insensitivity to high-power individuals when they behave more unfairly.

Several limitations of the present study should be acknowledged. First, the participants were relatively young, and compared to older people they may have more recently experienced low-power positions, for instance at school (with their teachers) or at work (with their supervisor). Greater familiarity with low-power positions could have led them to take more the perspective of the low-power character. Hence, the influence of age and work experience on the effect we have reported, should be examined in future research. However, the results of the Experiment 6 questionnaire showed that our participants were not particularly inclined to perceive themselves in a subordinate position, and our scenario did not involve a work-related situation.

Second, some aspects of the methodology we used might contribute to instill a feeling of powerlessness, such as the very fact of participating in a scientific experiment, which somehow places participants in a subject position, or the referential ambiguity conveyed by the question, which could create a feeling of uncertainty and anxiety. It could be worth to increasing the power position of participants to examine whether they are more likely to focus on the emotions of the high-power character. However, the self-reported questionnaire we used in Experiment 6 did not show any significant link between the perspective taken and participants' personal feeling of power.

Third, in the present research, we only investigated situations that elicit negative emotions and negative behavior, as the high-power individual clearly expressed her dissatisfaction with the low-power one. This could reinforce the weaker position of the lowpower character as compared to situations where the high-power character would behave more supportively towards the low-power character. Past works focusing on positive events such as fair sport competitions suggest that people also root for underdogs in these contexts 
(Vandello et al., 2007). It remains to test if such findings extend to all types of emotional ascriptions.

Finally, all our experiments were based on a paradigm where the referential ambiguity is relatively salient. Participants could thus be aware of the ambiguity and consciously choose between one or the other character. The possibility of a conscious choice could increase the inclination toward the subordinate character. Indeed, participants might think that it is socially more desirable to take the perspective of the low-power character than that of the high-power character. For instance, past research, using resource allocation and preference tasks in an inequality context, has shown that children favor more an advantaged individual over a disadvantaged individual when they are implicitly tested (Li, Spitzer \& Olson, 2014). It might therefore be useful in future research to explore other, more implicit, paradigms to address the issue investigated here. In order to make the choice between the two characters less salient, one possibility could be to remove the referential ambiguity and ask participants to list all the emotions the two characters may feel at the end of their interaction. In such a case, we would expect the emotions associated with the low-power character to be listed first. An alternative option could be to use the power interaction as a prime for target emotional stimuli. If participants take more the emotional perspective of the low-power character, the processing of emotional stimuli associated with that character should be facilitated.

Taken together, the findings reported here however reveal a clear inclination to privilege the emotional perspective of the powerless when attending to social interactions. While this inclination probably contributes to counterbalance inequalities and reinforce fairness within human groups, it might also lead to neglect what high-power individuals feel. This could bias the attitude of people involved in third-party actions such arbitrators, mediators or ombudspersons who might develop interventions rooted more on the lowpower's needs. 


\section{Acknowledgments}

The authors wish to thank very warmly the two actors Yvain Juillard (https://timeart.com/talent/yvain-juillard.htm) and Philippe Vindras for their dedicated contribution to the video-clips used in this study. This work was supported by the Labex/Idex CORTEX ANR11-LABX-0042, CNRS and INSERM. The authors declared that they had no conflicts of interest with respect to their authorship or the publication of this article.

\section{Author Contributions}

All authors contributed to the study concept, design, data collection and interpretation. F.Q, A.F. and E.C. performed the statistical analyses. F.Q. and J-B.VDH drafted the manuscript, and A.F., E.C., E.B. and Y.R. provided critical revisions. All authors approved the final version of the manuscript for submission. 


\section{References}

Abramovitch, R. (1976). The relation of attention and proximity to rank in preschool children. In M. R. A. Chance and R. R. Larsen (Eds.), The social structure of Ontogeny and social dominance Evolutionary Psychology - ISSN 1474-7049 - Volume 12 (pp. 153176). London: Wiley

Arnold, G., Sarlegna, F. R., Fernandez, L. G., \& Auvray, M. (2019). Somatosensory Loss Influences the Adoption of Self-Centered Versus Decentered Perspectives. Frontiers in psychology, 10.

Arnold, G., Spence, C., \& Auvray, M. (2016). Taking someone else's spatial perspective: Natural stance or effortful decentring?. Cognition, 148, 27-33.

Boroditsky, L., \& Ramscar, M. (2002). The roles of body and mind in abstract thought. Psychological Science, 13(2), 185-189. https://doi.org/10.1111/1467-9280.00434

Brescoll, V. L., \& Uhlmann, E. L. (2008). Can an angry woman get ahead? Status conferral, gender, and expression of emotion in the workplace. Psychological Science, 19(3), 268275. https://doi.org/10.1111/j.1467-9280.2008.02079.x

Breton, A., Cheylus, A., Baudouin, J. Y., \& Van der Henst, J. B. (2018). Status lies in the eye of the beholder: Evidence from eyetracking data. PsyArXiv. https://doi.org/10.17605/OSF.IO/R4Q3C

Chance, M. R. A., \& Larsen, R. R. (Eds.). (1976). The social structure of attention. London; New York: Wiley. 
Charafeddine, R., Mercier, H., Clément, F., Kaufmann, L., Berchtold, A., Reboul, A., \& Van der Henst, J.-B. (2015). How Preschoolers Use Cues of Dominance to Make Sense of Their Social Environment. Journal of Cognition and Development, 16(4), 587607.

Deaner, R. O., Khera, A. V., \& Platt, M. L. (2005). Monkeys pay per view : Adaptive valuation of social images by rhesus macaques. Current Biology, 15(6), 543548.

Enright, E. A., Alonso, D. J., Lee, B. M., \& Olson, K. R. (2020). Children's Understanding and Use of Four Dimensions of Social Status. Journal of Cognition and Development, 21(4), 573602.

Erle, T. M., \& Topolinski, S. (2015). Spatial and empathic perspective-taking correlate on a dispositional level. Social Cognition, 33, 187.

Erle, T. M., \& Topolinski, S. (2017). The grounded nature of psychological perspectivetaking. Journal of personality and social psychology, 112, 683.

Farroni, T., Menon, E., Rigato, S., \& Johnson, M. H. (2007). The perception of facial expressions in newborns. European Journal of Developmental Psychology, 4(1), 2-13.

Faul, F., Erdfelder, E., Lang, A.-G., \& Buchner, A. (2007). G*Power 3: A flexible statistical power analysis program for the social, behavioral, and biomedical sciences. Behavior Research Methods, 39, 175-191.

Fiske, S. T., \& Durante, F. (2019). Mutual status stereotypes maintain inequality. In The Social Psychology of Inequality (p. 335-348). Springer.

Fiske, S. T., \& Neuberg, S. L. (1990). A continuum of impression formation, from categorybased to individuating processes: Influences of information and motivation on attention and interpretation. Advances in Experimental Social Psychology, 23, 1-74 
Foulsham, T., Cheng, J. T., Tracy, J. L., Henrich, J. \& Kingstone, A. (2010). Gaze allocation in a dynamic situation: Effects of social status and speaking. Cognition, 117(3), 319-331.

Fragale, A. R., Overbeck, J. R., \& Neale, M. A. (2011). Resources versus respect : Social judgments based on targets' power and status positions. Journal of Experimental Social Psychology, 47(4), 767-775.

Freeman, J. B., Rule, N. O., Adams Jr, R. B., \& Ambady, N. (2009). Culture shapes a mesolimbic response to signals of dominance and subordination that associates with behavior. Neuroimage, 47, 353-359.

Galinsky, A. D., Magee, J. C., Inesi, M. E., \& Gruenfeld, D. H. (2006). Power and perspectives not taken. Psychological science, 17, 1068-1074.

Goldberg, L. R., Johnson, J. A., Eber, H. W., Hogan, R., Ashton, M. C., Cloninger, C. R., \& Gough, H. G. (2006). The international personality item pool and the future of publicdomain personality measures. Journal of Research in personality, 40, 84-96.

Gonzaga, G. C., Keltner, D., \& Ward, D. (2008). Power in mixed-sex stranger interactions. Cognition and Emotion, 22(8), 1555-1568.

Gülgöz, S., \& Gelman, S. A. (2017). Who’s the boss? Concepts of social power across development. Child development, 88(3), 946963.

Hall, J. A., Coats, E. J., \& LeBeau, L. S. (2005). Nonverbal behavior and the vertical dimension of social relations: a meta-analysis. Psychological bulletin, 131(6), 898.

Hanna, J. E., \& Tanenhaus, M. K. (2004). Pragmatic effects on reference resolution in a collaborative task: Evidence from eye movements. Cognitive Science, 28, 105-115. 
Hanna, J. E., Tanenhaus, M. K., \& Trueswell, J. C. (2003). The effects of common ground and perspective on domains of referential interpretation. Journal of Memory and Language, 49(1), 43-61.

Hareli, S., David, S., \& Hess, U. (2013). Competent and warm but unemotional: The influence of occupational stereotypes on the attribution of emotions. Journal of Nonverbal Behavior, 37(4), 307-317.

Hauser, D. J., Carter, M. S., \& Meier, B. P. (2009). Mellow Monday and furious Friday: The approach-related link between anger and time representation. Cognition and Emotion, 23(6), 1166-1180.

Heider, F., \& Simmel, M. (1944). An experimental study of apparent behavior. American Journal of Psychology, 57, 243-259.

Jeffreys, H. (1961). The theory of probability. Oxford UniversityPress.

Jones, B. C., Debruine, L. M., Main, J. C., Little, A. C., Welling, L. L., Feinberg, D. R., \& Tiddeman, B. P. (2010). Facial cues of dominance modulate the short-term gaze-cuing effect in human observers. Proceedings of the Royal Society B: Biological Sciences, 277(1681), 617-624.

Judd, C. M., Westfall, J., \& Kenny, D. A. (2012). Treating stimuli as a random factor in social psychology: A new and comprehensive solution to a pervasive but largely ignored problem. Journal of personality and social psychology, 103, 54.

Keltner, D., Gruenfeld, D. H., \& Anderson, C. (2003). Power, approach, and inhibition. Psychological Review, 110(2), 265. 
Kim, J., Allison, S. T., Eylon, D., Goethals, G. R., Markus, M. J., Hindle, S. M., \& McGuire, H. A. (2008). Rooting for (and then abandoning) the underdog. Journal of Applied Social Psychology, 38, 2550-2573.

Kitayama, S., Mesquita, B., \& Karasawa, M. (2006). The emotional basis of independent and interdependent selves: Socially disengaging and engaging emotions in the US and Japan. Journal of Personality and Social Psychology, 91(5), 890-903.

Li, V., Spitzer, B., \& Olson, K. R. (2014). Preschoolers reduce inequality while favoring individuals with more. Child development, 85(3), 1123-1133.

Magee, J. C., \& Smith, P. K. (2013). The social distance theory of power. Personality and Social Psychology Review, 17(2), 158-186.

Maner, J. K., DeWall, C. N., \& Gailliot, M. T. (2008). Selective attention to signs of success: Social dominance and early stage interpersonal perception. Personality and Social Psychology Bulletin, 34, 488-501.

Mattan, B. D., Rotshtein, P., \& Quinn, K. A. (2016). Empathy and visual perspective-taking performance. Cognitive neuroscience, 7, 170-181.

Michniewicz, K. S., \& Vandello, J. A. (2013). The attractive underdog: When disadvantage bolsters attractiveness. Journal of Social and Personal Relationships, 30(7), 942-952.

Nieuwland, M. S., \& Van Berkum, J. J. (2008). The neurocognition of referential ambiguity in language comprehension. Language and Linguistics Compass, 2(4), 603-630.

Over, H., \& Carpenter, M. (2015). Children infer affiliative and status relations from watching others imitate. Developmental science, 18(6), 917925. 
Pegna AJ, Khateb A, Lazeyras F, Seghier ML. (2005). Discriminating emotional faces without primary visual cortices involves the right amygdala, Nature Neurosciences, 8, 2425.

Pellegrini, A. D. (2008). The roles of aggressive and affiliative behaviors in resource control: A behavioral ecological perspective. Developmental Review, 28, 461-487.

Pun, A., Birch, S. A., \& Baron, A. S. (2016). Infants use relative numerical group size to infer social dominance. Proceedings of the National Academy of Sciences, 113(9), 23762381.

Quesque, F., Chabanat, E., \& Rossetti, Y. (2018). Taking the point of view of the blind: spontaneous level-2 perspective-taking in irrelevant conditions. Journal of experimental social psychology, 79, 356-364.

Ratcliff, N. J., Hugenberg, K., Shriver, E. R., \& Bernstein, M. J. (2011). The allure of status: High-status targets are privileged in face processing and memory. Personality and Social Psychology Bulletin, 37, 1003-1015.

Ridgeway, C. L. (2001). Gender, status, and leadership. Journal of Social issues, 57, 637-655.

Rouder, J. N., Speckman, P. L., Sun, D., Morey, R. D., \& Iverson, G. (2009). Bayesian t tests for accepting and rejecting the null hypothesis. Psychonomic bulletin \& review, 16(2), $225-237$.

Schönbrodt, F. D., Wagenmakers, E. J., Zehetleitner, M., \& Perugini, M. (2017). Sequential hypothesis testing with Bayes factors: Efficiently testing mean differences. Psychological Methods, 22(2), 322. 
Shamay-Tsoory, S. G., \& Aharon-Peretz, J. (2007). Dissociable prefrontal networks for cognitive and affective theory of mind: a lesion study. Neuropsychologia, 45(13), 30543067.

Shamay-Tsoory, S. G., Aharon-Peretz, J., \& Perry, D. (2009). Two systems for empathy: a double dissociation between emotional and cognitive empathy in inferior frontal gyrus versus ventromedial prefrontal lesions. Brain, 132(3), 617-627.

Terrizzi, B. F., Brey, E., Shutts, K., \& Beier, J. S. (2019). Children's developing judgments about the physical manifestations of power. Developmental psychology, 55(4), 793808.

Thomas, A. J., Thomsen, L., Lukowski, A. F., Abramyan, M., \& Sarnecka, B. W. (2018). Toddlers prefer those who win but not when they win by force. Nature Human Behaviour, 2,662669

Thomsen, L., Frankenhuis, W. E., Ingold-Smith, M. C., \& Carey, S. (2011). Big and mighty : Preverbal infants mentally represent social dominance. Science, 331(6016), 477.

Tiedens, L. Z., Ellsworth, P. C., \& Mesquita, B. (2000). Sentimental stereotypes: Emotional expectations for high-and low-status group members. Personality and Social Psychology Bulletin, 26(5), 560-575.

Tiedens, L. Z., \& Fragale, A. R. (2003). Power moves: complementarity in dominant and submissive nonverbal behavior. Journal of Personality and Social Psychology, 84(3), 558.

Vandello, J. A., Goldschmied, N. P., \& Richards, D. A. (2007). The appeal of the underdog. Personality and Social Psychology Bulletin, 33(12), 1603-1616. 
Vandello, J. A., Michniewicz, K. S., \& Goldschmied, N. (2011). Moral judgments of the powerless and powerful in violent intergroup conflicts. Journal of Experimental Social Psychology, 47(6), 1173-1178.

Van Kleef, G. A., Oveis, C., Van Der Löwe, I., LuoKogan, A., Goetz, J., \& Keltner, D. (2008). Power, distress, and compassion: Turning a blind eye to the suffering of others. Psychological science, 19, 1315-1322.

Wagner, D. G., \& Berger, J. (1997). Gender and interpersonal task behaviors: Status expectation accounts. Sociological perspectives, 40, 691.

Westfall, J., Judd, C. M., \& Kenny, D. A. (2015). Replicating studies in which samples of participants respond to samples of stimuli. Perspectives on Psychological Science, 10, 390399

Zitek, E. M., \& Tiedens, L. Z. (2012). The fluency of social hierarchy: the ease with which hierarchical relationships are seen, remembered, learned, and liked. Journal of Personality and Social Psychology, 102(1), 98. 